\title{
ESTUDIO DE LAS ISOTERMAS DE DESORCION DEL J ENGIBRE (Zingiber officinale)
}

Mari Medina $\mathrm{V}^{*}$

Oscar Mendieta T.*

\section{RESUMEN}

Para datos experimentales de humedad de equilibrio en desorción del jengibre, determinados entre 10 y $80 \%$ de humedad relativa, fueron evaluados 12 modelos matemáticos de isotermas a $278 \mathrm{~K}, 298 \mathrm{~K}$ y 318 K. La ecuación de D'arcy-Watt presentó el mejor grado de ajuste con coeficientes de determinación $\left(R^{2}\right)$ de 0,9999 para las tres temperaturas mencionadas. El cambio de energía libre y calor isotérico de desorción disminuyeron con el incremento en el contenido de humedad. La razón de calor latente de vaporización del agua en jengibre a calor latente de vaporización del agua libre decreció continuamente desde 1,5269 hasta 1,0446 en el rango de contenido de humedad de 6 a 18\%, y puede ser representada por un polinomio de tercer grado con un coeficiente de correlación de 0,9994 . Los valores obtenidos pueden ser utilizados para el diseño y control operacional del proceso de secado de este producto agrícola.

Palabras claves: J engibre, desorción, isotermas.

\section{INTRODUCCION}

El jengibre es una especie de alto valor económico, muy conocida en la selva peruana, cuya producción puede ser incentivada en la región San Martín pues cuenta con tierras aptas para este cultivo; siendo una de las principales formas de conservación de este producto agrícola, el secado o deshidratación.

\footnotetext{
* Facultad de Ingeniería Agroindustrial - Universidad Nacional de San Martín. Casilla
} 
El contenido de humedad de equilibrio es una importante propiedad de los materiales que tiene un impacto significativo en el manipuleo, procesamiento y almacenamiento de todos los materiales higroscópicos, Hutchinson, D y Otten, L. (1984). Se define contenido de humedad de equilibrio como el contenido de humedad del material después de haber sido expuesto a un determinado ambiente por un período muy largo de tiempo. El mecanismo que controla este fenómeno está gobernado por la presión de vapor de agua. Una representación gráfica de contenido de humedad de equilibrio del material versus humedad relativa del ambiente es conocida comúnmente como isoterma de adsorción o desorción, Shepherd, H. y Bhardwaj, R. (1986).

Numerosas investigaciones han sido efectuadas para determinar el contenido de humedad de equilibrio de una gran variedad de productos higroscópicos. Diversas ecuaciones teóricas, semiteóricas y empíricas han sido desarrolladas y utilizadas en el análisis de datos de contenido de humedad de equilibrio, Hutchinson, D y Otten, L. (1984). Boquet, Iglesias y Chirife (1978) presentaron una extensa revisión de ecuaciones que describen las isotermas de desorción aplicables a productos alimentarios.

Los objetivos del presente estudio fueron:

- Evaluar diferentes ecuaciones de isotermas de desorción para valores experimentales de humedad de equilibrio y humedad relativa obtenidos en la desorción del jengibre.

- Determinar el cambio de energía libre, calor isotérico de desorción y calor latente de vaporización para jengibre.

\section{MATERIALES Y METODOS}

Los valores experimentales de humedad de equilibrio y humedad relativa para la desorción del jengibre a 278 K, 298 K y 318 K fueron tomados de Sinicio y Roa (1979).

Las ecuaciones de isotermas empleadas se presentan en el Cuadro 1. Estas ecuaciones fueron ajustadas a los valores experimentales mediante análisis de regresión, lineal o no lineal según el caso, utilizando el paquete estadístico Statgraf 4.0.

El grado de concordancia entre los valores experimentales y los predecidos por las diferentes ecuaciones fue verificado mediante el coeficiente de determinación $\left(R^{2}\right)$ y el error medio relativo (RMS\%), calculado este último según la ecuación: 


\section{Cuadro № 1}

\section{Modelos matemáticos de isotermas de desorción}

№ $\quad$ NOMBRE DEL MODELO

\section{ECUACION}

(1) Smith

$M=K_{1}-K_{2} \operatorname{Ln}(1-A w)$

(2) Bradley

$M=\operatorname{Ln}: \operatorname{Ln}(1 / A w) / K_{2}: / \operatorname{Ln} K_{1}:$

(3) Oswin

$M=K_{1}: A w /(1-A w): K_{2}$

(4) Caurie

$M=K 1 \operatorname{EXP}\left(-K_{2} A w\right)$

(5) Harkins-J ura

$M=:\left(K_{2}-\operatorname{Ln} A w\right) / K_{1}::^{0.5}$

(6) Bet

$$
\frac{A w}{(1-A w) M}=\frac{1}{K_{1} K_{2}}+\frac{K_{2}-1}{K_{1}-K_{2}} w
$$

(7) Chung-Pfost

$$
M=\frac{-1}{K_{2}} \operatorname{Ln} \frac{T \operatorname{Ln} A w}{-K_{1}}
$$

(8) Halsey

$$
M=\operatorname{EXPK}_{1}\left(1 / K_{2}\right)
$$

-Ln Aw

(9) Henderson-

$$
\operatorname{Ln}(1-A w) \quad\left(1 / K_{2}\right)
$$

Thompson

$M=$

(10) Chen-Clayton

$$
M=\frac{-K_{1}^{-K_{1}}}{K_{2}} \operatorname{Ln} \stackrel{\operatorname{Ln~Aw}}{-K_{1}}
$$

(11) Gab

$\frac{\mathrm{AW}}{\mathrm{AW^{2 }}=}+\begin{array}{ll}1 & \mathrm{~K}_{2}{ }^{2}-\mathrm{K}_{1} \mathrm{~K}_{2}{ }^{2} \\ \mathrm{AW}+\end{array}$
$M$
$\mathrm{K}_{1} \mathrm{~K}_{2} \mathrm{~K}_{3}$
$\mathrm{K}_{1} \mathrm{~K}_{2} \mathrm{~K}_{3}$
$\mathrm{K}_{1} \mathrm{~K}_{2} \mathrm{~K}_{3}$

(12) D'arcy-Watt

$$
\begin{aligned}
& \mathrm{K}_{1} \mathrm{~K}_{2} \text { Aw } \\
& \mathrm{K}_{3} \mathrm{~K}_{4} \mathrm{Aw} \\
& M= \\
& +\mathrm{K}_{5} \mathrm{Aw}+ \\
& 1+\mathrm{K}_{1} \mathrm{Aw} \\
& 1-\mathrm{K}_{3} \mathrm{Aw}
\end{aligned}
$$


RMS\% $=100 ? \overline{\text { (Meq. exp. - Meq. sim.) } / \text { Meq. exp. }\left.\right|^{2}}$

$\mathrm{N}$

en donde $\mathrm{N}$ es el número de datos.

La presión de vapor de saturación del agua libre (Pvs) para cada temperatura fue determinada con la ecuación (14) (Pvs en $\mathrm{N} / \mathrm{m}^{2}$ ) :

Pvs $=\operatorname{EXP}\left(60,43-\frac{6834,27}{T+273}-5,17 \operatorname{Ln}(T+273)\right)$

El valor de la presión de vapor (Pv) para cada temperatura, fue calculado según la ecuación:

$$
\mathrm{PV}=\mathrm{PVS} * \mathrm{HR}
$$

La humedad relativa para la ecuación anterior fue calculada a través de la ecuación que describió mejor los puntos experimentales para valores de humedad de equilibrio comprendidos entre 6 y 18\% (b.h.).

El cambio de energía libre para las diferentes temperaturas consideradas fue obtenido de la ecuación :

$$
? \mathrm{~F}=\mathrm{R} T \operatorname{Ln}(\mathrm{Pv} / \mathrm{Pvs})
$$

Las constantes de la ecuación (17) que relaciona ?F y contenido de humedad fueron determinadas mediante regresión lineal (mínimos cuadrados) para las tres temperaturas consideradas en el estudio.

$$
-? F=-K_{1} \operatorname{EXP}\left(-K_{2} M\right)
$$

Para el cálculo del calor isotérico de desorción se utilizó la ecuación:

$$
-? \mathrm{H}=\mathrm{R} \quad \frac{\mathrm{T}_{1} \mathrm{~T}_{2}}{\mathrm{~T}_{2}-\mathrm{T}_{1}} \operatorname{Ln} \frac{\mathrm{P}_{2}}{\mathrm{P}_{1}}
$$


y para el calor neto de desorción :

$$
q=? H-2
$$

Mediante regresión lineal se determinó la razón calor latente de vaporización del agua en jengibre a calor latente de vaporización del agua libre (L/Ls) de la ecuación de Othmer (5):

$$
\operatorname{Ln}(P \vee)=\frac{L}{L s} \operatorname{Ln}(P \vee s)+C
$$

\section{RESULTADOS Y DISCUSION}

Las constantes de los doce modelos matemáticos evaluados se presentan en el Cuadro 2. De las ecuaciones utilizadas la que mejor reprodujo los valores experimentales fue la ecuación de D'arcy-Watt, con valores del coeficiente de determinación $\left(R^{2}\right)$ de 0,9999 para las tres temperaturas de trabajo. En los tres casos el error medio relativo (RM S\%) fue inferior al 1\%. Según Saravacos et al (1986) esta ecuación puede ser aplicada para actividades de agua comprendidas entre 0,10 y 0,90; sin embargo la necesidad de estimar cinco parámetros puede hacer a este modelo menos atractivo para su aplicación en alimentos. Los valores experimentales y las curvas ajustadas con esta ecuación se presentan en la Figura 1. 
FIG. I ISOTERMAS DE DESORCICN DE JENGIBRE

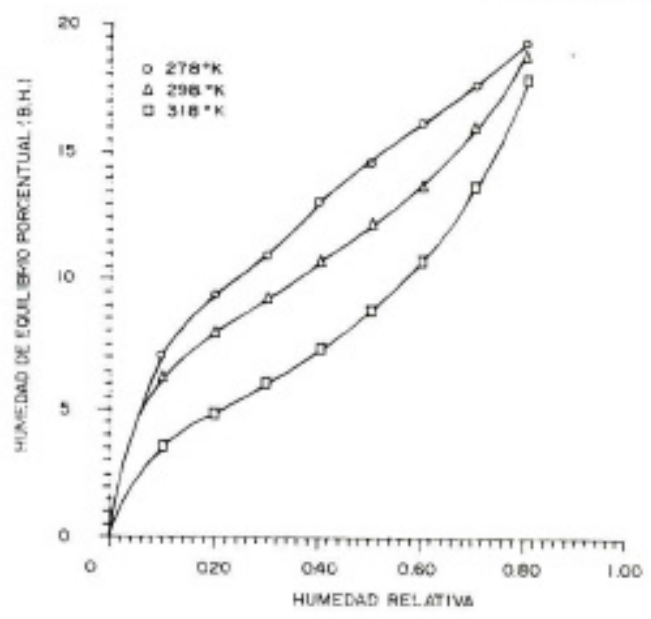

FIG. 2 CAMEIO EN ENERGIA LIERE DE DESORCION PARA JENGIBRE

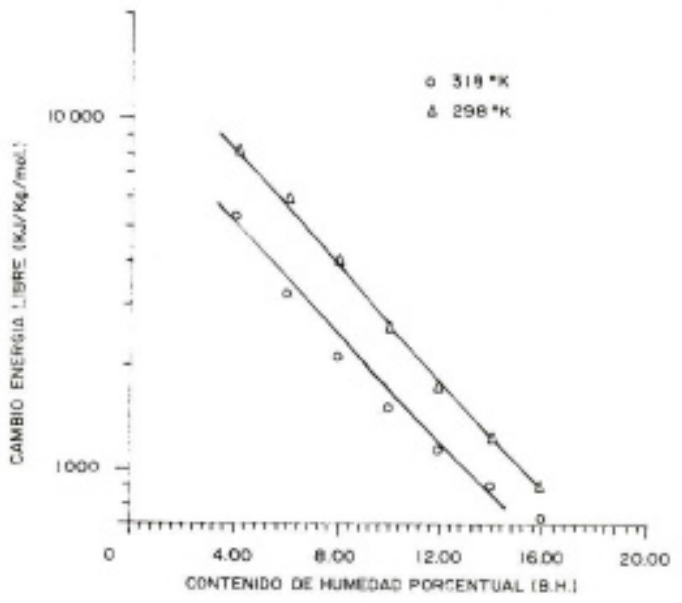




\section{Cuadro 2}

\section{Valores de las Constantes de las Isotermas Evaluadas para J engibre}

\begin{tabular}{|c|c|c|c|c|c|c|c|c|}
\hline \multirow[t]{2}{*}{ Ecuación } & \multirow{2}{*}{$\begin{array}{r}\text { Tem } \\
\text { (으) }\end{array}$} & \multicolumn{5}{|c|}{-------------------- Constantes ------------------ } & \multirow{2}{*}{$\begin{array}{c}\text { Coef. Det. } \\
\mathrm{R}^{2}\end{array}$} & \multirow[t]{2}{*}{ RMS\% } \\
\hline & & $\mathrm{K}_{1}$ & $\mathrm{~K}_{2}$ & $\mathrm{~K}_{3}$ & $\mathrm{~K}_{4}$ & $\mathrm{~K}_{5}$ & & \\
\hline \multirow[t]{3}{*}{ Smith } & 278 & 8,0679 & 7,9378 & & & & 0,9946 & 10,4290 \\
\hline & 298 & 6,2263 & 8,2298 & & & & 0,9992 & 4,4294 \\
\hline & 318 & 2,5401 & 9,4154 & & & & 0,9995 & 2,6807 \\
\hline \multirow[t]{3}{*}{ Bradley } & 278 & 0,9306 & 9,6033 & & & & 0,9854 & 3,9936 \\
\hline & 298 & 0,8321 & 6,9344 & & & & 0,9960 & 2,6436 \\
\hline & 318 & 0,8492 & 3,4072 & & & & 0,9673 & 13,3859 \\
\hline \multirow{3}{*}{ Oswin } & 278 & 14,1046 & 0,2631 & & & & 0,9984 & 5,1026 \\
\hline & 298 & 12,3475 & 0,3132 & & & & 0,9999 & 1,2021 \\
\hline & 318 & 9,0946 & 0,4859 & & & & 0,9994 & 4,8785 \\
\hline \multirow[t]{3}{*}{ Caurie } & 278 & 7,5534 & $-1,2301$ & & & & 0,9974 & 7,9418 \\
\hline & 298 & 5,8894 & $-1,4605$ & & & & 0,9996 & 2,8665 \\
\hline & 318 & 2,9206 & $-2,2473$ & & & & 0,9991 & 3,7945 \\
\hline Harkins- & 278 & 180,8967 & 0,2256 & & & & 0,9977 & 7,5402 \\
\hline \multirow[t]{2}{*}{ J ura } & 298 & 113,1693 & 0,0826 & & & & 0,9997 & 3,0766 \\
\hline & 318 & 45,2635 & $-0,0888$ & & & & 0,9972 & 11,0168 \\
\hline \multirow[t]{3}{*}{ Bet } & 278 & 5,2705 & $-4,92 \mathrm{E} 13$ & & & & 0,9277 & 26,9574 \\
\hline & 298 & 4,4680 & $6,61 \mathrm{E05}$ & & & & 0,9537 & 24,7214 \\
\hline & 318 & 3,9307 & 248,7522 & & & & 0,9924 & 10,3420 \\
\hline \multirow[t]{3}{*}{ Bett** } & 278 & 7,8518 & 52,1068 & & & & 0,9715 & 4,0014 \\
\hline & 298 & 6,4564 & 102,4204 & & & & 0,9803 & 3,0981 \\
\hline & 318 & 4,6856 & 19,9306 & & & & 0,9984 & 1,0489 \\
\hline Chung- & 278 & 2669,46 & 0,1856 & & & & 0,9854 & 4,1125 \\
\hline \multirow[t]{2}{*}{ Pfost } & 298 & 2066,02 & 0,1838 & & & & 0,9960 & 2,6471 \\
\hline & 318 & 1083,42 & 0,1635 & & & & 0,9673 & 13,3883 \\
\hline \multirow[t]{3}{*}{ Halsey } & 278 & 5,7944 & 2,3639 & & & & 0,9363 & 8,3050 \\
\hline & 298 & 4,9510 & 2,1500 & & & & 0,9893 & 3,5848 \\
\hline & 318 & 2,7601 & 1,4499 & & & & 0,9960 & 3,2456 \\
\hline Henderson- & 278 & $5,59 \mathrm{E}-04$ & 2,6637 & & & & 0,9982 & 1,3756 \\
\hline \multirow[t]{2}{*}{ Thompson } & 298 & $1,18 \mathrm{E}-03$ & 2,5071 & & & & 0,9848 & 4,1656 \\
\hline & 318 & 0,0147 & 1,7068 & & & & 0,9731 & 8,1213 \\
\hline Chen- & 278 & 9,6032 & 0,1856 & & & & 0,9854 & 3,9935 \\
\hline \multirow[t]{2}{*}{ Clayton } & 298 & 6,9344 & 0,1838 & & & & 0,9960 & 2,6436 \\
\hline & 318 & 3,4072 & 0,1634 & & & & 0,9673 & 13,3859 \\
\hline \multirow[t]{3}{*}{ Gab } & 278 & 12,3387 & 20,5052 & 0,5147 & & & 0,9996 & 2,0379 \\
\hline & 298 & 8,2319 & 31,7377 & 0,7230 & & & 0,9999 & 0,8097 \\
\hline & 318 & 5,3007 & 14,9318 & 0,8935 & & & 0,9999 & 1,2841 \\
\hline D' arcy- & 278 & 29,0485 & 7,4703 & $-0,0541$ & 1,0503 & 15,4157 & 0,9999 & 0,7727 \\
\hline \multirow[t]{2}{*}{ Watt } & 298 & 46,6498 & 6,4633 & 0,9032 & 2,3816 & 8,2370 & 0,9999 & 0,4584 \\
\hline & 318 & 14,3327 & 5,2686 & 0,8199 & 8,1685 & $-2,9639$ & 0,9999 & 0,7303 \\
\hline
\end{tabular}

** Para valores de Aw entre $0,1-0,5$ 
La ecuación Gab presentó el segundo mejor grado de ajuste para las tres temperaturas consideradas con valores del error medio relativo iguales o inferiores al $2 \%$. Este modelo de adsorción semiteórico ha sido considerado como el mejor modelo para muchos materiales alimenticios, Mok, et al. (1990). Las demás ecuaciones evaluadas presentaron menor grado de ajuste.

El cambio de energía libre viene a ser la energía requerida para transferir moléculas del estado de vapor a la superficie del sólido o de la superficie del sólido al estado de vapor, y puede ser considerado como una medida del trabajo efectuado por el sistema al llevarse a cabo los procesos de adsorción y desorción. El cambio de energía libre decreció continuamente con el incremento en el contenido de humedad. fue:

La relación entre energía libre y contenido de humedad, obtenida

para $278 \mathrm{~K}$ :

$$
\begin{gathered}
-? F=321033 \times 10^{3} \operatorname{EXP}(-0,1685 M) \\
R=-0.995
\end{gathered}
$$

para $298 \mathrm{~K}$ :

$$
\begin{gathered}
-? F=1391696 \times 10^{3} \operatorname{EXP}(-0,1823 M) \\
R=-0,998
\end{gathered}
$$

para 298 :

$$
\begin{gathered}
-? F=1439779 \times 10^{3} \operatorname{EXP}(-0,1760 \mathrm{M}) \\
R=-0,989
\end{gathered}
$$

El ploteo de las ecuaciones (22) y (23) es mostrado en la Figura 2.

El calor de desorción es la cantidad de calor requeridos para la migración de humedad cuando ésta desorbe como vapor. Shepherd y Bhardwaj (1986) indican que es una medida del calor o energía que debe ser adicionada al adsorber gas para romper la fuerza intermolecular existente entre las moléculas de vapor de agua y la superficie del adsorbente. En el Cuadro 3 se presentan los valores de calor isotérico y calor neto de desorción calculados con las ecuaciones (18) y (19). Puede apreciarse que el calor de desorción decrece con el aumento en el contenido de humedad desde 90,62 hasta 45,21 MJ $/ \mathrm{kg} \mathrm{mol}$. 


\section{Cuadro 3}

Calor isotérico de desorción y calor neto de desorción para jengibre

\begin{tabular}{lcccc}
\hline $\begin{array}{l}\text { Humedad de } \\
\text { equilibrio } \\
\text { (\% b.h.) }\end{array}$ & \multicolumn{2}{c}{$\begin{array}{c}\text { Calor isotérico de } \\
\text { desorción } \\
\text { (MJ / kg mol) }\end{array}$} & \multicolumn{2}{c}{$\begin{array}{c}\text { Calor neto de } \\
\text { desorción } \\
(\mathrm{MJ} / \mathrm{kg} \mathrm{mol})\end{array}$} \\
\hline & $288 \mathrm{~K}$ & $308 \mathrm{~K}$ & $288 \mathrm{~K}$ & $308 \mathrm{k}$ \\
\hline 6,0 & 47,8948 & 90,6157 & 46,7617 & 87,9778 \\
8,0 & 56,8171 & 76,7162 & 55,6840 & 74,0783 \\
10,0 & 60,3468 & 63,0273 & 59,2137 & 60,3894 \\
12,0 & 57,8286 & 54,8806 & 56,6955 & 52,2427 \\
14,0 & 53,5562 & 50,1675 & 52,4231 & 47,5296 \\
16,0 & 49,7703 & 47,2090 & 48,6372 & 44,5711 \\
18,0 & 46,8763 & 45,2055 & 45,7432 & 42,5676 \\
\hline
\end{tabular}

El ploteo de la ecuación de Othmer (20) para contenidos de humedad constantes comprendidos entre 6 y 18\% dio un juego de líneas rectas (isóteras) cuyas pendientes corresponden a la razón L/Ls. El coeficiente de correlación de las isóteras en el ploteo de la ecuación de Othmer fue superior al 0,995. La pendiente de las isóteras decreció en forma continua con el incremento en el contenido de humedad como se muestra en la Figura 3.

Para la razón L/Ls (pendientes de Othmer) y el contenido de humedad se obtuvo una relación dada por un polinomio de tercer grado de la forma siguiente:

$$
\begin{gathered}
\frac{L}{L S}=1,25372+0,11708 \mathrm{M}-0,01427 \mathrm{M}^{2}+0,000396 \mathrm{M}^{3} \\
R=0,9994
\end{gathered}
$$

El ploteo de la ecuación (24) es mostrado en la Figura 4.

Valores de calor latente de vaporización para contenidos de humedad comprendidos entre 6 y 18\%, a las temperaturas de $278 \mathrm{~K}$ y $318 \mathrm{~K}$, se presentan en el Cuadro 4. Puede observarse que el calor latente de vaporización del agua en jengibre decrece continuamente con el incremento en el contenido de humedad y la razón L/Ls tiende a la unidad conforme el contenido de humedad del jengibre se incrementa. 
FIG. 3 RECTAS DE OTHMER PARA JENGIBRE

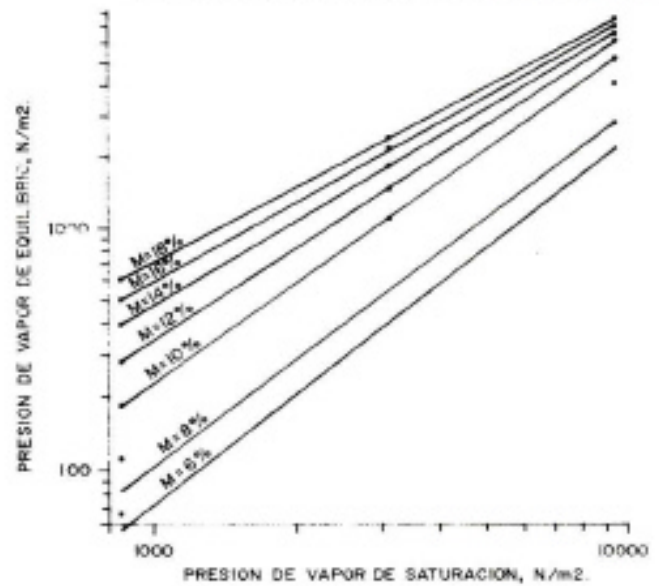

FIG. 4 VARIACION DEL CALOR LATENTE DE VAPORIZACION DEL. AGUA DE JENGIBRE

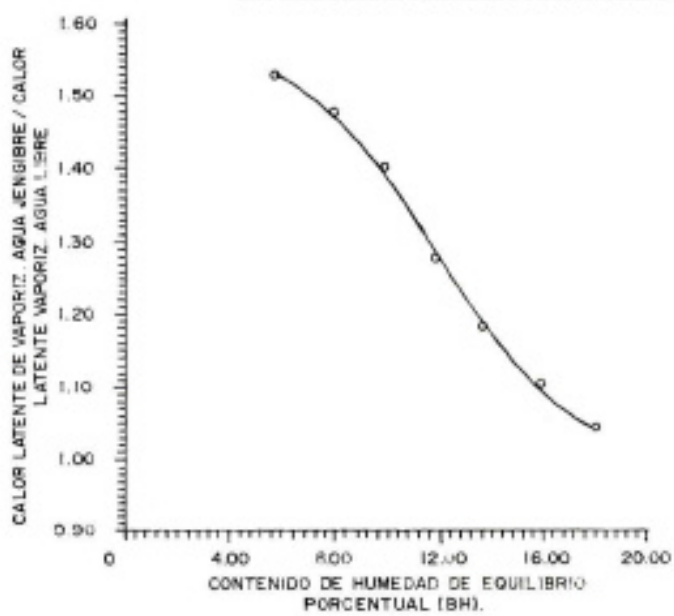




\section{Cuadro 4}

Calor latente de vaporización del agua de jengibre

\begin{tabular}{|c|c|c|c|c|}
\hline \multirow{2}{*}{$\begin{array}{l}\text { Humedad de } \\
\text { equilibrio } \\
\text { (\% b.h.) }\end{array}$} & \multicolumn{3}{|c|}{ Calor latente de vaporización } & \multirow[t]{2}{*}{ L/LS } \\
\hline & $278 \mathrm{~K}$ & $298 \mathrm{~K}$ & $318 \mathrm{~K}$ & \\
\hline 6,0 & 3801,60 & 3729,45 & 3656,77 & 1,5269 \\
\hline 8,0 & 3720,68 & 3650,07 & 3578,94 & 1,4944 \\
\hline 10,0 & 3478,68 & 3412,66 & 3346,15 & 1,3972 \\
\hline 12,0 & 3192,11 & 3131,53 & 3070,50 & 1,2821 \\
\hline 14,0 & 2939,40 & 2883,62 & 2827,42 & 1,1806 \\
\hline 16,0 & 2746,69 & 2694,57 & 2642,05 & 1,1032 \\
\hline 18,0 & 2600,79 & 2551,44 & 2501,71 & 1,0446 \\
\hline Agua & 2489,75 & 2442,50 & 2394,90 & 1,0000 \\
\hline
\end{tabular}

\section{CONCLUSIONES}

1. La ecuación de D'arcy-Watt describió las isotermas de desorción del jengibre con un alto coeficiente de correlación y mínimo valor del error medio relativo, sobre todo el rango de temperatura y humedad considerado.

2. El calor isotérico de desorción, el calor neto de desorción y el cambio de energía libre decrecieron en forma continua con el incremento del contenido de humedad.

3. El calor latente de vaporización del agua en jengibre decrece con el incremento en el contenido de humedad y un polinomio de tercer grado correlaciona la razón L/Ls con el contenido de humedad. 


\section{NOMENCLATURA UTILIZADA}

\section{Símbolo}

\section{Definición}

Calor isotérico de desorción.

Humedad relativa, decimal.

$\mathrm{K}_{1}, \mathrm{~K}_{2}, \mathrm{~K}_{3}$,

$\mathrm{K}_{4}, \mathrm{~K}_{5}$

L

Ls

M

Meq. exp.

Meq. sim.

$P_{v}$

Pvs

$P_{1}, P_{2}$

R

$\mathrm{T}$

$T_{1}, T_{2}$

? F

$?$

de isotermas. agua en jengibre. agua libre. porcentaje en base húmeda porcentaje en base húmeda. porcentaje en base húmeda.

Presión de vapor del agua en jengibre.

Temperatura.

Cambio en energía libre.
Constantes de las ecuaciones

Calor latente de vaporización del

$\mathrm{kJ} / \mathrm{k}$

Calor latente de vaporización del

$\mathrm{kJ} / \mathrm{kg}$

Contenido de humedad del jengibre,

Contenido de humedad experimental,

Contenido de humedad calculado,

$\mathrm{N} / \mathrm{m}^{2}$

Presión de vapor de saturación.

$\mathrm{N} / \mathrm{m}^{2}$

Presiones de vapor de equilibrio a temperaturas $T_{1}$ y $T_{2}$, respectivamente.

$\mathrm{N} / \mathrm{m}^{2}$

Constante universal de los gases.

$\mathrm{J} / \mathrm{kg} \mathrm{mol} \mathrm{K}$

K

Temperaturas de isotermas adyacentes.

K

$\mathrm{kJ} / \mathrm{kg} \mathrm{mol}$

Calor latente de vaporización del agua a una temperatura especificada
$\mathrm{kJ} / \mathrm{kg}$ 


\section{BIBLIOGRAFIA}

BOQUET, R.; CHIRIFE, J. and IGLESIAS, H. 1978. "Equations for fitting water sorption isotherms of foods: II. Evaluation of varius two parameter models", en J. Fd. Technol. 13:319-327.

HUTCHINSON, D. y OTTEN, L. 1984. "Equilibrium Moisture Content of White Beans", en: Cereal Chemistry 61(2): 155-158.

MOK, C. y HETTIARACHCHY, N. 1990. "Moisture Sorption Characteristics of Ground Sunfllowert Nutemeat and its Products", en J. Fd. Sci. 55(3): 786-789.

ROSSI, S.; FIOREZE, R.; DE SOUZA, V. y RAMALHO, H. 1981. "Curvas e Equaçoes de Equilibrio Higroscópico para 15 Produtos Agrícolas, en Rev. braz. de armaz., Vicosa, 6(2):5-10.

SARAVACOS, G.; TSIOURVAS, D. y TSAMI, E. 1986. "Effect of Tempera ture on the water adsorption isotherms of Sultana Raisins", en J. Fd. Sci. 51(2): 381-383, 387.

SHEPHERD, H. y BHARDWAJ , R. 1986. "A Study of the desorption isotherms of rewetted pigeon pea Type-17", en J. Fd Sci. 51(3): 595-598.

SINICIO, R. y ROA, G. 1979. "Curvas de Teor de Equilibrio e Calor latente de Vaporizaçao para Castanhas de Caju e Raspas de Mandioca", en: Rev. Braz. de armaz.; Viçosa, 4(2): 45-55. 